\title{
HIGH-PERFORMANCE CAPACITANCE-TO-DIGITAL CONVERTERS FOR SUB-NM DISPLACEMENT SENSING: A REVIEW
}

\author{
Hui Jiang ${ }^{1}$ AND Stoyan Nihtianov ${ }^{1,2^{*}}$ \\ ${ }^{1}$ Delft University of Technology, \\ Electronic Instrumentation Laboratory, \\ Mekelweg 5, 2628 CD Delft, The Netherlands, \\ e-mail: H.Jiang@tudelft.nl \\ ${ }^{2}$ Technical University of Sofia, \\ 8, St. Kliment Ohridski Blvd, 1756 Sofia, Bulgaria, \\ e-mail:S.Nihtianov@tudelft.nl
}

\begin{abstract}
This paper presents a review of energy-efficient capacitance-to-digital converters specifically intended for precision displacement measurement. Apart from achieving good energy efficiency, such capacitance-to-digital converters must achieve high accuracy and stability, as well as high immunity to power-supply and common-mode variations. Various capacitance-to-digital converter architectures are discussed, beginning with traditional designs in which a capacitance-to-voltage converter is used to drive an analog-to-digital converter, and moving on to more recent works which attempt to increase energy efficiency and reduce complexity by eliminating the capacitance-to-voltage converter. The performance of these topologies, and in particular their energy efficiency, will be compared and summarized. Moreover, a novel capacitance-to-digital converter topology for sub-nm displacement sensing with an electrically floating target is described.

Keywords: capacitance-to-digital converter, energy efficiency, precision displacement measurement, capacitance-to-voltage converter, sub-nm, electrically floating target.
\end{abstract}

\section{INTRODUCTION}

Capacitive displacement sensors, ideally, generate no noise and consume no static power, making them very suitable for energy-constrained applications, ranging from advanced industrial machines, which require minimum selfheating errors, to smartphones and Internet of Things (IoT) gadgets, which

${ }^{*}$ Corresponding author.

DOI: 10.7546/EngSci.LVII.20.02.02

Engineering Sciences, LVII, 2020, No. 2 
need a proliferation of battery power [1-20]. Capacitive displacement sensors offer the advantages of a simple structure, low cost, and excellent performance. However, the readout approach is difficult, as it is usually based on the AC modulation of the electric field of the sensor, which is affected by a variation in the physical parameter of interest. On the other hand, to realize the capacitive sensing system in an energy-efficient, robust, and accurate manner, the electronics readout must also be energy-efficient, robust, and accurate [9].

Over the years, most efforts have involved improving the energy efficiency of the capacitive sensor readout. Figure 1(a) shows a conventional capacitive sensor readout which consists of a Capacitance-to-Voltage Converter (CVC) followed by an Analog-to-Digital Converter (ADC). The CVC converts the capacitance output to a voltage level that is large enough to drive the succeeding ADC [10]. In Figure 1 we show a square wave AC excitation signal, since using harmonic (sinusoidal) excitation in older solutions has always resulted in higher power consumption. As a first stage, the CVC defines the performance of the overall readout in terms of noise and power consumption. However, maintaining accuracy requires the ADC to be as good as the CVC, leading to an area- and power-hungry design for both stages. An alternative is a direct digitization readout approach (CDC), which is becoming increasingly popular as it eliminates the CVC stage, as shown in Fig. 1(b). The motivation for this approach is the elimination of the CVC and the associated high-gain amplifiers, which results in lower complexity and reduced IC area.

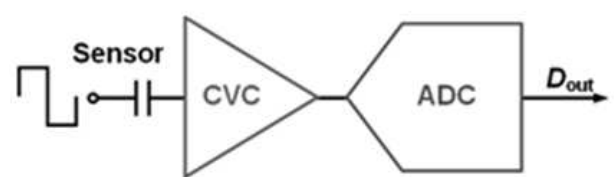

(a)

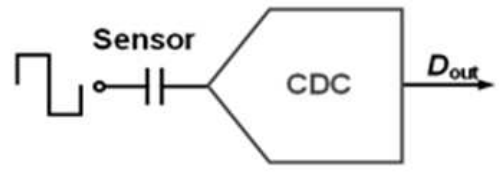

(b)

Fig. 1. (a) Conventional capacitive sensor readout;

(b) a capacitance-to-digital converter

This paper presents a review of energy-efficient Capacitance-to-Digital Converters (CDCs) intended for displacement sensing, and a novel CDC design. The paper is organized as follows. Section II discusses different CDC architectures for displacement sensing. Section III introduces a CDC with electrically floating target sensing capability and its application. Comparisons of stateof-art CDCs are given in Section IV. Finally, the conclusion is drawn. 


\section{DIFFERENT CAPACITANCE-TO-DIGITAL CONVERTERS}

Capacitance-to-digital converters can be designed using a wide variety of architectures: Successive-Approximation-Register (SAR) [3, 4, 13, 14], sigmadelta $(\Sigma \Delta)[2,5-7,11,12]$, period/pulse-width modulation (PM/PWM) $[8,9$, 20], and single/multi-slope [13, 15-19], depending on the target specifications. For instance, the SAR topology can be suitable for low- to medium-resolution applications, which is often limited by quantization noise, with impressively low power consumption. On the other hand, $\Sigma \Delta$ CDCs can achieve high resolution with relatively high power consumption. One of the fundamental reasons for this is that the $\Sigma \Delta \mathrm{CDC}$ requires a large oversampling ratio (OSR) to reduce the in-band quantization noise and thermal noise to achieve high resolution.

In many papers on precision CDCs for displacement sensing, the power efficiency figure of merit $(F o M)$ is used as an indication of their relative performance, which is given by [20]:

$$
\text { FoM }=\frac{\text { Power } \times T_{\text {measurement }}}{2^{E N O B}},
$$

where $E N O B$ is the effective number of bits based on the dynamic range $(D R)$ in $\mathrm{dB}, T_{\text {measurement }}$ is the conversion time, and Power is the power dissipation. $D R$ is given by:

$$
D R=20 \log \left(\frac{C_{\text {range }} / \sqrt{2}}{C_{\text {rms }}}\right) .
$$

Most CDCs intended for sub-nm resolution displacement sensing have a $\mu \mathrm{m}$ $D R$, corresponding to a capacitance range $C_{\text {range }}$ of a few $\mathrm{pF}$. They require an RMS noise level $C_{r m s}$ in the atto-Farad (aF) range, with a bandwidth of up to a few kHz. This often results in a DR of about $90 \mathrm{~dB}$ and $E N O B$ of 15 bits. Figure 2 shows a comparison of state-of-the-art CDCs in terms of energy efficiency.

Some recently published results have broken the wall of the $1 \mathrm{pJ} /$ conversion step $F o M$ successfully. Especially, the $\Delta \Sigma$ CDC can achieve a reasonable $E N O B$ precision displacement sensing applications which is higher than 15 bits. However, as shown in Fig. 3, the challenge becomes much evident when state-of-the-art CDCs are evaluated differently. From the resolution point of view, all of the above-mentioned CDCs, except $\Delta \Sigma$ CDCs and PM CDCs, are one order away from aF-level resolution. Providing such high resolution is a non-trivial task. Generally, most CDCs are realized based on corresponding ADCs, inheriting some advantages and disadvantages. Making the right choice 


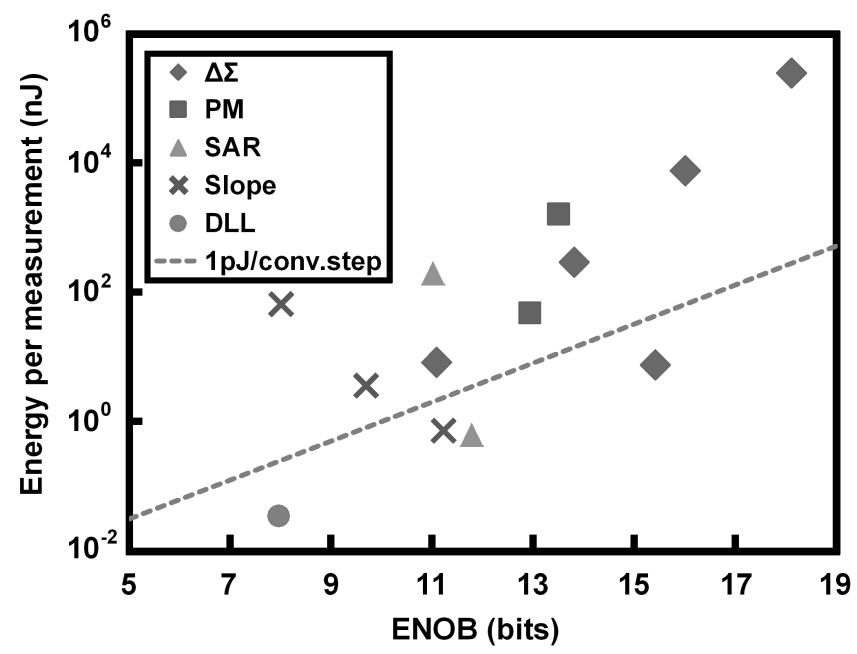

Fig. 2. Energy consumption versus $E N O B$ of published CDCs based on the $\Delta \Sigma$ modulator $(\Delta \Sigma)$, period modulator ADC (PM), successive approximation ADC (SAR), slope ADC (Slope) and digital locked loop (DLL)

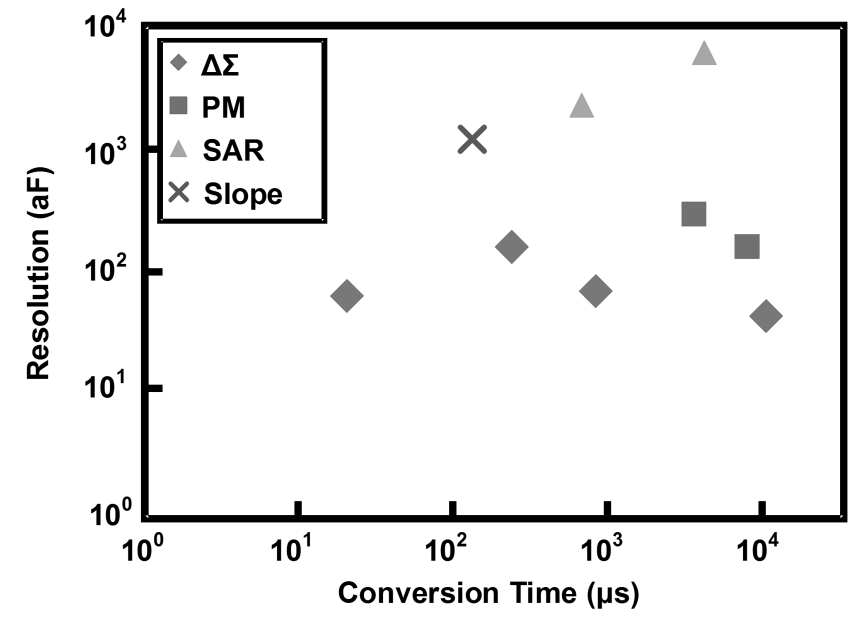

Fig. 3. Capacitance resolution versus conversion time of published CDCs based on the $\Delta \Sigma$ modulator $(\Delta \Sigma)$,

Period Modulator ADC (PM), SAR, slope

ADC (Slope) and digital locked loop (DLL)

depends on the different applications targeted. In this section, different CDCs, their advantages, and disadvantages will be discussed. 


\subsection{SAR CDC}

A SAR CDC is derived from the architecture of a SAR ADC, which is one of the most energy efficient ADCs. It is an undisputed fact that the SAR CDC can achieve high energy efficiency in some applications [3]. As Figure 4 shows, the low power dissipation benefit offered by SAR CDCs comes from their zero-static power building blocks [4]. All circuits in this SAR CDC work dynamically. The capacitive sensor is connected to the Capacitive Digitalto-Analog Converter (CDAC). The measured capacitance is converted to a voltage at the input of the comparator. Here the CDAC and the capacitive sensor are combined, resulting in the direct conversion of variable capacitance to digital output.

The SAR CDC operates in two phases. The first phase is the sample phase. During this phase, the capacitive sensor is charged by a DC voltage source, $k V_{r e f}$, while the CDAC is simultaneously charged by a voltage source. This is the DC constant voltage approach. During the conversion phase, the capacitive sensor is connected to the CDAC to redistribute the charge saved on the sensor and CDAC. After the charge redistribution, the capacitive sensor is biased in the constant charge approach. The capacitance value of the sensor will be quantized by switching the bottom of the CDAC, which is controlled by SAR logic, without consuming any static current.

Low power, fast conversion, and simple architecture are the main advantages of the SAR CDC. However, there are also several disadvantages:

1) The baseline capacitance of the sensor will "eat up" the effective dynamic range of the SAR CDC because of the charge redistribution. During the conversion phase, the capacitance of the sensor, including baseline capacitance and variable capacitance, is converted to a digital signal. This is because the baseline capacitance value is always much larger than the variable one to compensate for the baseline capacitance value of the sensor. As shown in Fig. $4, C_{m}$ is used to cancel the effect of the baseline capacitance of the sensor by using the zoom-in technique. However, these two $C_{m}$ work as two parasitic capacitors at the input of the comparator reducing the input voltage swing of the comparator significantly. Thus the ENOB of the SAR CDC is limited. A SAR ADC with a Correlated Double Sampling (CDS) front-end can solve this problem [13].

2) It is difficult to improve the capacitance resolution by reducing the quantization step. There are two options to further improve the capacitance resolution by using SAR architecture. One is to design a SAR CDC with a smaller unit capacitor and low noise comparator. However, in this case, the total $\mathrm{kT} / \mathrm{C}$ noise charge will be dominant. Furthermore, a low noise 


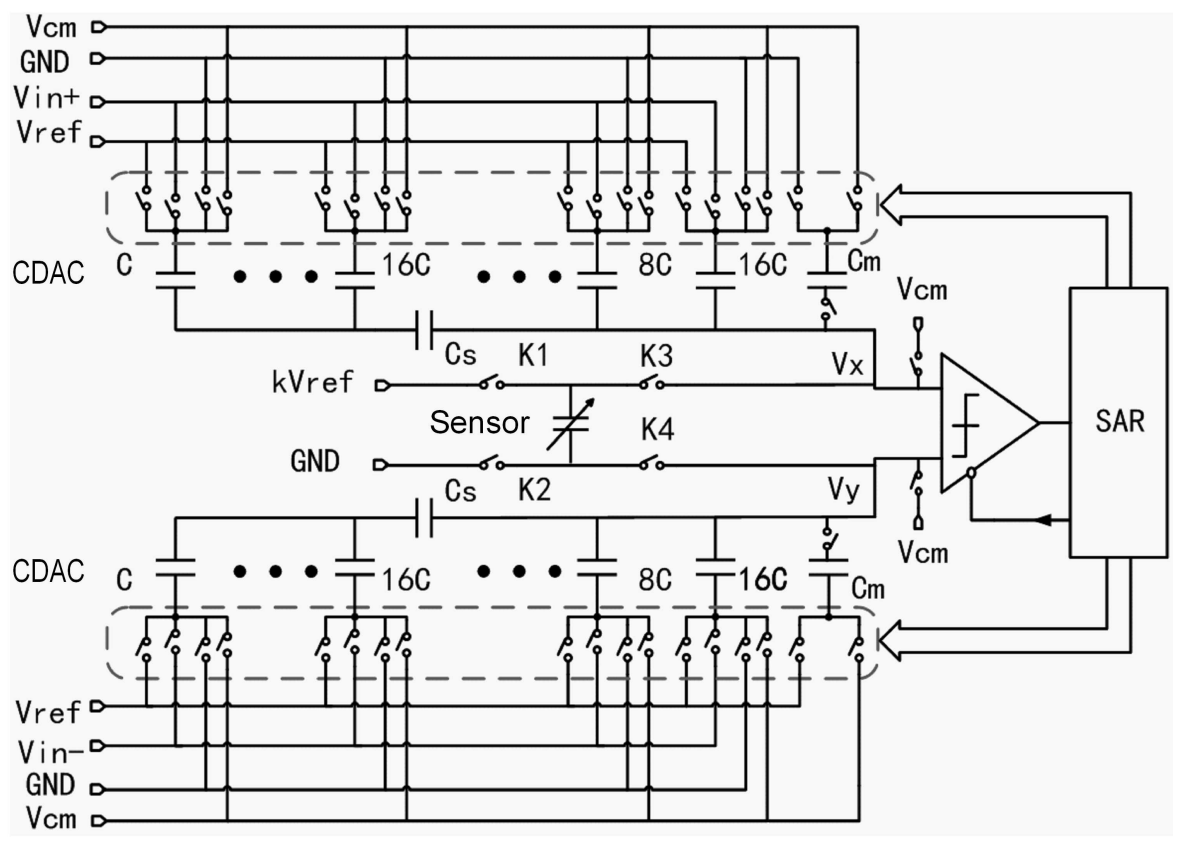

Fig. 4. Typical SAR CDC [4]

comparator will burn much more power than the dynamic one. The second option is to use oversampling to obtain a lower quantization noise in a CDC. However, in practice, the CDAC mismatch will limit the DR. Without digital calibration, the SAR CDC can achieve a 12 bit ENOB [4]. Moreover, oversampling will increase the $T_{\text {measurement }}$ and thus increase energy consumption.

\section{2. $\Delta \Sigma \mathrm{CDC}$}

With oversampling and noise shaping, a $\Delta \Sigma$ ADC can achieve a very high $E N O B$. Its resolution and linearity do not rely on component matching because of the ideal linearity of the 1 bit DAC. Most $\Delta \Sigma$ CDCs are realized using a discrete-time (DT) $\Delta \Sigma$ ADC architecture. In Figure 5, the core block of a single-bit quantizer $\Delta \Sigma \mathrm{CDC}$ is shown with one integrator and one comparator [11]. A capacitive sensor is embedded into the $\Delta \Sigma$ modulator as a sampling capacitor. The capacitive sensor is biased in a similar way to AC constant voltage [9]. Due to the negative feedback of the loop, the charge which is proportional to the value of the capacitive sensor, $C_{x}$, will be balanced by the charge which is proportional to the value of the reference capacitance, 
$C_{r e f}$. Then $C_{x}$ can be precisely expressed by the density, $\mu=C_{x} / C_{r e f}$, of the bitstream, which is generated by the $\Delta \Sigma$ modulator.

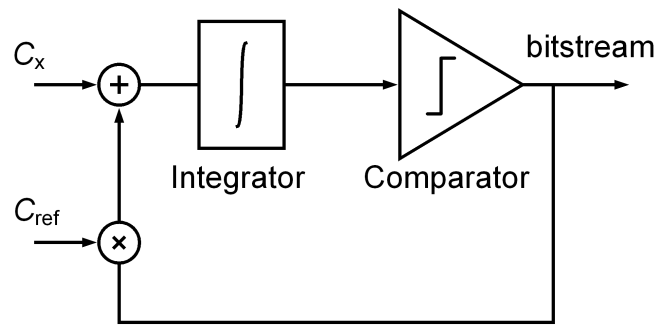

Fig. 5. First-order $\Delta \Sigma \mathrm{CDC}$

The advantages of DT $\Delta \Sigma$ CDCs are high resolution with moderately complex circuitry. They can become much faster by increasing the order of the $\Delta \Sigma$ modulator loop. However, there are also some limitations:

1) The kTC noise usually dominates the noise performance of DT $\Delta \Sigma$ CDCs since most DT $\Delta \Sigma$ CDCs are implemented using Switched Capacitor (SC) circuits. To reach aF-level resolution, a sufficient oversampling ratio is needed. Thus, the $T_{\text {measurement }}$ of $\Delta \Sigma$ CDCs will become much higher than SAR CDCs.

2) During the integrating phase of the SC integrator, it will take more time for the output of the integrator to reach the final state because of the exponential settling behaviour. To ensure accurate settling, the sampling clock period has to be long enough compared to the time constant of the charge transfer process [20]. This results in a higher bandwidth requirement, and therefore, higher power consumption for the SC integrator OTA.

\subsection{Slope CDC}

As shown in Fig. 6, the value of the capacitance is transferred to a corresponding time interval by the integrator of the CDCs. This time interval is then digitized by a counter. Most slope CDCs are realized using the architecture of the integrating ADC. Thus slope CDCs enjoy the advantages of integrating ADCs, which are well known for their simple yet high resolution. However, slope CDCs, like integrating ADCs, also suffer from low conversion speed or high power consumption resulting from the subsequent counter, since the counter requires $2 n$ clocks to realize $n$-bit data.

Multi-slope architecture can be utilized to reduce $T_{\text {measurement }}$. However, improving the linearity of the multi-slope CDCs over the entire capacitance range requires additional power. The output headroom of the integrator is 

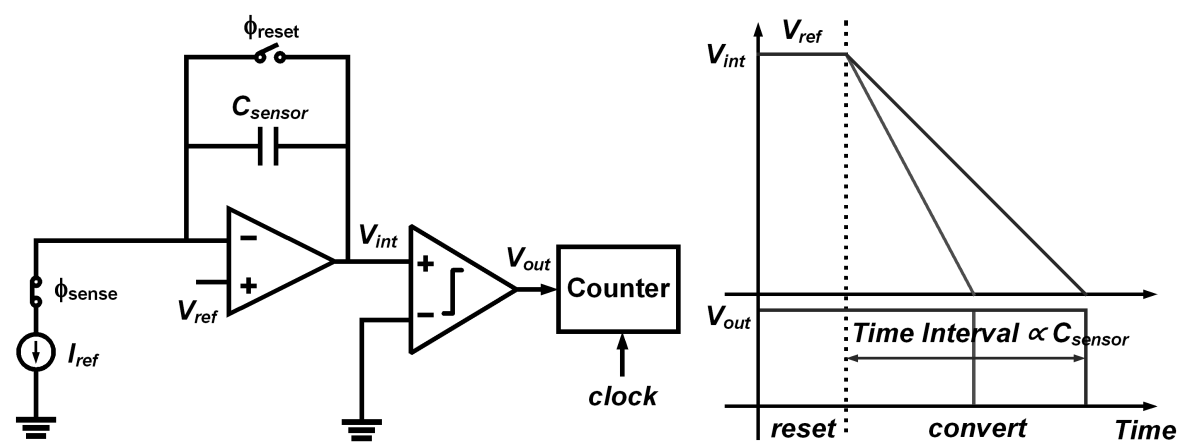

Fig. 6. Slope CDC

also an issue. In an ideal case, the integrator of the slope CDC should have a class $\mathrm{AB}$ output stage or use negative feedback to regulate the swing of the output signal of the integrator [9].

\subsection{Period modulator CDC}

Period modulator (PM) CDCs, as shown in Fig. 7, are essentially relaxation oscillators. The value of capacitance is firstly converted to a period. Then, the period is digitized by the following counter [8]. PM CDCs have almost all the advantages of single slope CDCs: They are compact and can achieve high resolution. However, they also have similar disadvantages, namely a slow conversion speed and a power-hungry counter.

In this section, the major features of CDCs for displacement sensing are reviewed. The performance of most CDCs for precision displacement sensing is limited by $\mathrm{kT} / \mathrm{C}$ noise and measurement time. Table 1 compares the CDCs

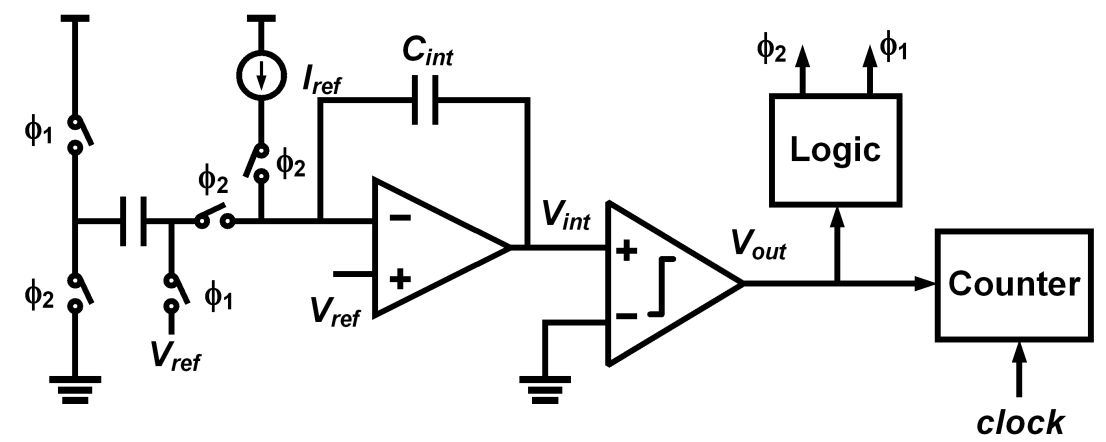

Fig. 7. PM CDC 
Table 1. Comparison of CDCs

\begin{tabular}{|c|c|c|c|c|}
\hline Type & SAR CDC & $\Delta \Sigma$ CDC & Slope CDC & PM CDC \\
\hline Power & ++ & + & - & - \\
\hline$T_{\text {measurement }}$ & ++ & + & - & - \\
\hline Resolution & - & ++ & + & + \\
\hline
\end{tabular}

in terms of power, measurement time, and resolution. As indicated in Table $1, \Delta \Sigma$ CDCs are the most promising candidates for sub-nm level displacement sensing applications.

\section{FULLY CONTACTLESS $\Delta \Sigma$ CDC}

Capacitive displacement sensors are potentially more energy efficient than eddy-current sensors. However, one major drawback compared to eddy-current sensors is that conventional CDCs operate by connecting the target in a closedloop signal configuration, as shown in Fig. 8(a) [5-8]. This means that the target electrode must be connected to the same ground as the interface electronics [21], which prevents the capacitive sensor from being fully contactless. What is more, the ground loop incorporating the target and the input of the electronic interface is susceptible to electromagnetic interference and acts as a source of parasitic capacitance, both of which degrade the sensor performance.

To minimize the loop while offering fully contactless displacement sensing, readouts with electrically floating targets have been proposed [10] Fig. 8(b). The sensor readout employs two identical capacitive sensing electrodes, positioned close to the target electrode. This results in two capacitors in series $\left(C_{s 1}=C_{s 2}=C_{s}\right)$ whose magnitudes equally vary with the distance, $d$, to the target. By exciting the capacitors with anti-phase voltages $\pm V_{s}$, the value of $C_{s}$ can be obtained by measuring the charge $Q_{s}$. Compared to the conventional capacitive readout approach, this significantly reduces the area of the sensing loop and thus minimizes the error caused by the interfering magnetic fields [5]. However, such readouts with electrically floating targets are relatively power-hungry compared to conventional CDCs [10]. Moreover, any mismatch between the two sensing electrodes degrades the readout CMRR and thus its electric field interference immunity. To reduce the error caused by the misalignment of the probe and the target, the CDC needs a multi-channel input to obtain an out-of-plane tilt angle [22].

In practical implementations, the parasitic capacitance between the floating target and its surrounding environment $\left(C_{i p}\right.$ up to $\left.500 \mathrm{fF}\right)$ introduces a major challenge. It forms a path for interfering electric fields, primar- 


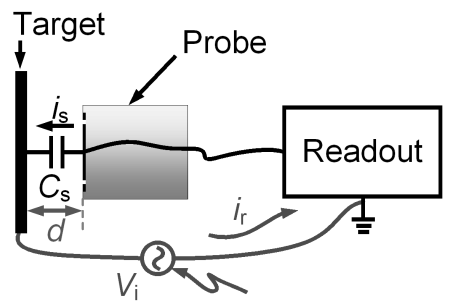

(a)

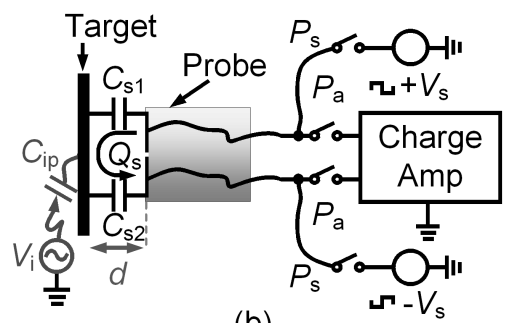

(b)

Fig. 8. (a) Conventional capacitive displacement sensing system; (b) contactless capacitive displacement sensing with a floating target

ily created by mains lines $(50 / 60 \mathrm{~Hz})$. As shown in Fig. 8(b), the sensor front-end consists of a differential charge integrator with switched capacitor excitation. $C_{s 1}$ and $C_{s 2}$ are periodically charged, delivering a signal charge $Q_{s}=\left(C_{s 1} C_{s 2}\right)\left(C_{s 1}+C_{s e}\right) V d d$ to the integrator. An interfering signal $V_{i}$ then couples to the target electrode via $C_{i p}$, resulting in a common-mode signal that will be rejected by the differential charge integrator.

However, a mismatch between the sensing electrodes $\left(C_{s 1} \neq C_{s 2}\right)$, due to fabrication errors and/or alignment errors $(\theta)$, results in a deteriorated CMRR [10]. It is shown in [10] that even with mismatched electrodes, a high CMRR is achieved by chopping the signal charge $Q_{i}$ and the integrating capacitors $C_{\text {inta }}$ and $C_{\text {intb }}$ (see Fig. 9). The input switches $P_{1}-P_{3}$ are controlled by the chopping signal $P_{c h}$ preserving the integration operation for the $C_{s}$ measurement. Due to the action of the input chopper, $C_{\text {inta }}$ and $C_{\text {intb }}$ continuously swap their position, such that $C_{\text {inta }}$ always accumulates negative charge $\left(Q_{s a}\right)$, while $C_{i n t b}$ always accumulates positive charge $\left(Q_{s b}\right)$. At the same time, the mismatched interference charges $\left(Q_{i 1}\right.$ and $\left.Q_{i 2}\right)$, as well as the offset and the $1 / f$ noise of the integrator, are up-modulated to the out-of-band frequencies, which will be filtered in the digital domain.

As shown in Fig. 10, the CDC is based on a second-order feedforward 1bit $\Delta \Sigma$ modulator. The modulator sampling frequency $f_{s}$ is $1 \mathrm{MHz}$, which is enough to achieve the target resolution with an OSR of 1000. The chopping frequency is set at $10 \mathrm{kHz}$ to minimize quantization noise fold-back [23]. The differential signal charge $Q_{s}$ is counterbalanced by a feedback DAC, in which $C_{f b}=10 \mathrm{pF}$. Depending on the output bitstream, it delivers a charge of $\pm C_{f b} V_{d d} / 2$. This charge-balancing results in an output bitstream with an average value of $C_{s} / C_{f b}$. To maximize the use of the CDC available dynamic range, the modulator uses a programmable 5-bit trimming DAC to cancel the baseline capacitance, where $C_{T-\max }=31 \mathrm{pF}$. 

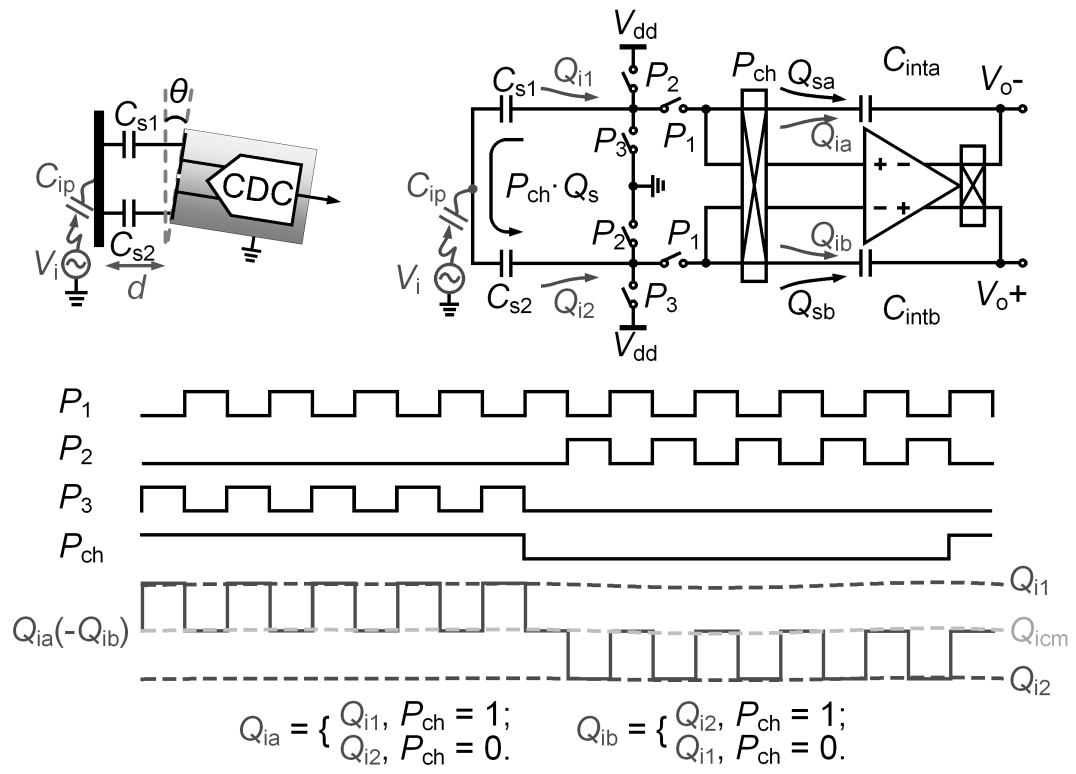

Fig. 9. Differential charge integrator with mismatched electrodes

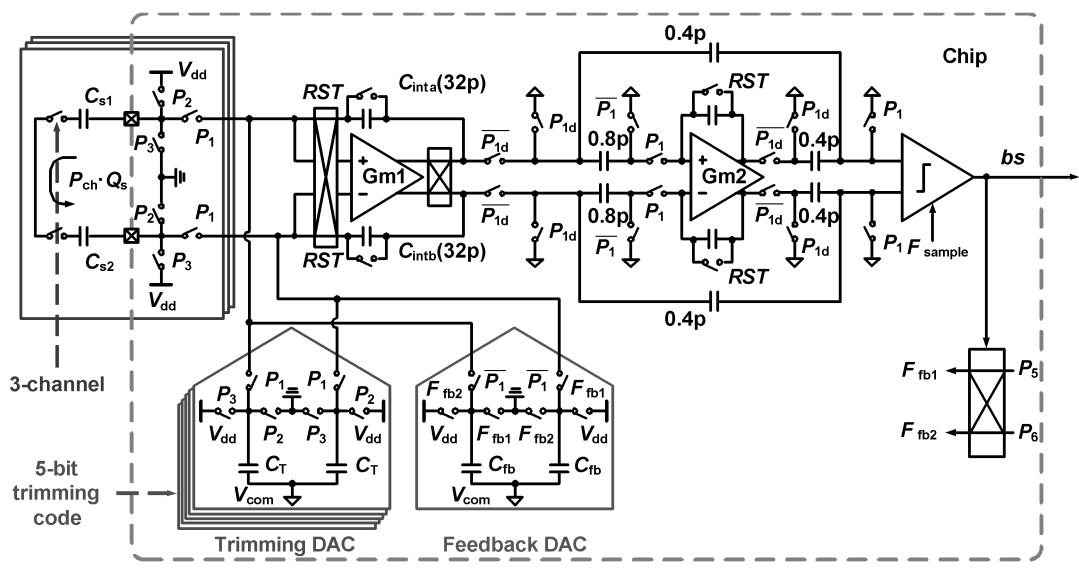

Fig. 10. Block diagram of the proposed three-channel CDC

Table 2 summarizes the performance of the CDC and the state-of-the-art. Compared to conventional CDCs [5-8], the proposed CDC offers floating target sensing capability, while achieving similar energy efficiency. 
Table 2. Performance summary and comparison

\begin{tabular}{|c|c|c|c|c|c|c|}
\hline & This work & {$[5]$} & {$[6]$} & {$[7]$} & {$[8]$} & {$[9]$} \\
\hline Technology $(\mu \mathrm{m})$ & 0.18 & 0.35 & 0.35 & 0.16 & 0.16 & - \\
\hline Floating target & Yes & No & No & No & No & Yes \\
\hline Power $(\mathrm{mW})$ & 0.56 & 14.9 & 0.76 & 0.003 & 0.014 & $121^{*}$ \\
\hline Cap-range $(\mathrm{pF})$ & $0-10$ & $8-12$ & $6-22$ & $0-3.8$ & $0-8$ & $0-10$ \\
\hline SNR $(\mathrm{dB})$ & 98.5 & 84.8 & 102.6 & 114.6 & 80.9 & 81.3 \\
\hline Conversion $(\mathrm{ms})$ & 1 & 0.02 & 10.5 & 100 & 6.86 & 0.1 \\
\hline FoM(pJ/Conv.-step) & 8.0 & 20.9 & 74 & 0.76 & 10.4 & $369^{*}$ \\
\hline
\end{tabular}

${ }^{*}$ Without considering the power consumption of the ADC.

\section{CONCLUSION}

In this paper, a review of energy efficient CDCs intended for displacement sensing is given. Various CDC architectures are discussed. Among them, $\Delta \Sigma \mathrm{CDCs}$ are the promising candidates for sub-nm level displacement sensing applications. The CDC embeds the push-pull principle in a second-order $\Sigma \Delta$ modulator. Experimental results show that it achieves a 98.5 dB SNR within a $1 \mathrm{~ms}$ conversion time. This CDC has an in-band CMRR of higher than 117 dB and achieves a comparable resolution FoM while offering floating target sensing capability. Compared to other ultra-low-power CDCs, the CDCs for displacement sensing have less energy efficiency, due to multiple trade-offs between energy-efficiency and other important parameters such as baseline capacitance, stability and linearity. However, the energy efficiency gap is not that large and so will probably be bridged by future CDC designs.

\section{REFERENCES}

[1] O. S. van de Ven, J. G. Vogel, S. Xia, J. W. Spronck, and S. Nihtianov, Self-Aligning and Self-Calibrating Capacitive Sensor System for Displacement Measurement in Inaccessible Industrial Environments, IEEE Transactions on Instrumentation and Measurement (Feb. 2018) 67 (2) 350-358.

[2] S. Oh, W. Jung, K. Yang, D. Blaauw, and D. Sylvester, 15.4b Incremental Sigma-Delta Capacitance-to-Digital Converter with Zoom-in 9b Asynchronous SAR, in: Digest of Symposium on VLSI Circuits, June 2014, pp. 222223.

[3] S. Hussaini, H. Jiang, P. Walsh, D. MacSweeney, and K. A. A. MakINWA, A 15-nW per Sensor Interference-Immune Readout IC for Capacitive Touch Sensors, IEEE Journal of Solid-State Circuits (July 2019) 54 (7) 18741882 . 
[4] H. Jiang, Z. Wang, C. Zhang, H. Jiang, And Z. Wang, A combined low power SAR capacitance-to-digital analog-to-digital converter for multisensory system, Analog Integrated Circuits and Signal Processing (May 2013) 75 (2) $311-322$.

[5] S. Xia, K. A. A. Makinwa, and S. Nihtianov, A capacitance-to-digital converter for displacement sensing with $17 \mathrm{~b}$ resolution and $20 \mu$ s conversion time, in: ISSCC Digest Technical Papers, Feb. 2012, pp. 197-198.

[6] R. Yang, M. A. P. Pertijs, and S. Nihtianov, A Precision Capacitanceto-Digital Converter with 16.7-bit ENOB and 7.5-ppm $/{ }^{\circ} \mathrm{C}$ Thermal Drift, IEEE Journal of Solid-State Circuits, (Nov. 2017) 52 (11) 3018-3031.

[7] B. Yousefzadeh, W. Wu, B. Buter, K. Makinwa, and M. Pertijs, A compact sensor readout circuit with combined temperature, capacitance and voltage sensing functionality, in: Digest of Symposium on VLSI Circuits, Kyoto, 2017.

[8] Y. He, Z. Chang, L. Pakula, S. H. Shalmany, and M. Pertijs, A $0.05 \mathrm{~mm}^{2} 1 \mathrm{~V}$ capacitance-to-digital converter based on period modulation, in: Digest of IEEE International Solid-State Circuits Conference (ISSCC), San Francisco, CA, USA, 2015.

[9] A. Heidary and G. C. M. Meijer, Features and Design Constraints for an Optimized SC Front-End Circuit for Capacitive Sensors With a Wide Dynamic Range, IEEE Journal of Solid-State Circuits (July 2008) 43 (7) 1609-1616.

[10] X. Guo and S. N. Nihtianov, A capacitive sensing technique for measuring displacement with one floating target electrode, in: Digest of 2010 IEEE International Conference on Industrial Technology, Mar. 2010, pp. 1565-1570.

[11] Z. Tan, R. DaAmen, A. Humbert, Y. Ponomarev, Y. Chae, and M. A. P. Pertiss, A 1.2-V 8.3-nJ CMOS Humidity Sensor for RFID Applications, IEEE J. Solid-State Circuits (Oct. 2013) 48 (10) 2469-2477.

[12] Z. Tan, S. H. Shalmany, G. C. M. Meijer, and M. A. P. Pertijs, An energy-efficient 15-bit capacitive-sensor interface based on period modulation, IEEE J. Solid-State Circuits (Jul. 2012) 47 (7) 1703-1711.

[13] H. Ha, D. Sylvester, D. Blaauw, and J. Sim, A 160nW 63.9fJ/conversionstep Capacitance-to-Digital Converter for Ultra-Low-Power Wireless Sensor Nodes, in: ISSCC Digest Technical Papers, Feb. 2014, pp. 220-221.

[14] H. Omran, M. Arsalan, and K. N. Salama, A Robust Parasitic-Insensitive Successive Approximation Capacitance-to-Digital Converter, in: IEEE Proceedings of CICC, San Jose, CA, USA, Sept. 2014.

[15] H. Omran, M. Arsalan, and K. N. Salama, 7.9 pJ/Step Energy-Efficient Multi-Slope 13-bit Capacitance-to-Digital Converter, IEEE Transactions on Circuits and Systems II: Express Briefs (Aug. 2014) 61 (8) 589-593. 
[16] S. Oh, Y. Lee, J. Wang, Z. Foo, Y. Kim, D. Blaauw, and D. Sylvester, Dual-Slope Capacitance to Digital Converter Integrated in an Implantable Pressure Sensing System, in: IEEE ESSCIRC Digest Technical Papers, Sept. 2014, pp. $295-26$.

[17] N. T. Trung And P. Häfliger, A Submicrowatt Implantable Capacitive Sensor System for Biomedical Applications, IEEE Transactions on Circuits and Systems II: Express Briefs (Feb. 2015) 61 (8) 209-213.

[18] W. Jung, S. Jeong, S. Oh, D. Sylvester, and D. Blaauw, A 0.7 pFto-10nF fully digital capacitance-to-digital converter using iterative delay-chain discharge, in: ISSCC Digest Technical Papers, Feb. 2015, pp. 484-485.

[19] K. Tanaka, Y. Kuramochi, T. Kurashina, K. Okada, and A. MatSUZAWA, A $0.026 \mathrm{~mm}^{2}$ capacitance-to-digital converter for biotelemetry applications using a charge redistribution technique, in: IEEE ASSCC Digest Technical Papers, Nov. 2007, pp. 244-247.

[20] Z. TAN, Energy-Efficient Capacitive-Sensor Interfaces, Ph.D. Dissertation, Delft University of Technology, Delft, the Netherlands (2013).

[21] M. R. Nabavi, M. A. P. Pertijs, and S. Nihtianov, An Interface for EddyCurrent Displacement Sensors With 15-bit Resolution and $20 \mathrm{MHz}$ Excitation, in IEEE Journal of Solid-State Circuits (Nov. 2013) 48 (11) pp. 2868-2881.

[22] H. Jiang, S. Amani, J. G. Vogel, S. H. Shalmany, and S. Nihtianov, A 117-dB In-Band CMRR 98.5-dB SNR Capacitance-to-Digital Converter for Sub-nm Displacement Sensing with an Electrically Floating Target, in IEEE Solid-State Circuits Letters (2020) 3 9-12.

[23] H. Jiang, C. Ligouras, S. Nihtianov, and K. A. A. Makinwa, A 4.5 $\mathrm{nV} / \sqrt{ } \mathrm{Hz}$ Capacitively Coupled Continuous-Time Sigma-Delta Modulator with an Energy-Efficient Chopping Scheme, in IEEE Solid-State Circuits Letters (Jan. 2018) 1 (1) 18-21.

Received April 16, 2020 